\title{
Rupture of non-communicating rudimentary pregnant uterine horn in a pseudo-unicornuate uterus with placenta increta at 27 weeks of amenorrhea
}

\author{
Moulay Elmehdi EI Hassani ${ }^{1,}$, , Houda Fagouri ${ }^{1}$, Abdellah Babahabib ${ }^{1}$, Hicham Bakkali ${ }^{2}$, \\ Jaouad Kouach ${ }^{1}$, Driss Moussaoui Rahali ${ }^{1}$, Mohamed Dehayni ${ }^{1}$ \\ ${ }^{1}$ Department of Obstetrics and Gynaecology, Military Hospital of Rabat, CHU Ibn Sina, Rabat, Morocco \\ ${ }^{2}$ Department of Anesthesia, Military Hospital of Rabat, CHU Ibn Sina, Rabat, Morocco
}

\section{Email address:}

docteurelhassani@gmail.com (M. E. El Hassani), houdhoudf14@yahoo.fr (H. Fagouri), babahabib2003@hotmail.com (A. Babahabib), hbakkali@ymail.com (H. Bakkali), jaouad_kouach@yahoo.fr (J. Kouach), moussaouidriss@yahoo.fr (D. M. Rahali), mohamedehayni@gmail.com (M. Dehayni)

\section{To cite this article:}

Moulay Elmehdi El Hassani, Houda Fagouri, Abdellah Babahabib, Hicham Bakkali, Jaouad Kouach, Driss Moussaoui Rahali, Mohamed Dehayni. Rupture of Non-Communicating Rudimentary Pregnant Uterine Horn in a Pseudo-Unicornuate Uterus with Placenta Increta at 27 Weeks of Amenorrhea. Journal of Gynecology and Obstetrics. Vol. 2, No. 5, 2014, pp. 77-80. doi: 10.11648/j.jgo.20140205.13

\begin{abstract}
Pregnancy in a non-communicating rudimentary horn is an extremely rare usually resulting in rupture during second trimester of pregnancy. The standard treatment is excision of the pregnancy and rudimentary horn. We report a case of ruptured non-communicating rudimentary horn at 27 weeks in a woman with previous vaginal delivery. She presented to emergency with acute abdominal pain and massive hemoperitoneum. Excision of the rudimentary horn was done at laparotomy and the patient had an uneventful recovery.
\end{abstract}

Keywords: Non-Communicating Rudimentary Uterine Horn, Pregnancy, Uterine Rupture, Placenta Increta

\section{Introduction}

A unicornuate uterus results from the normal differentiation of only one mullerian duct. Various subtypes of unicornuate uterus present $5 \%$ of all the congenital anomalies and are one of the least common malformations. More frequently, they do have a rudimentary horn which doesn't communicate to the contralateral uterus. These non-communicating rudimentary horns contain a functional endometrial cavity in $55 \%$ and do not have a cervix or a vaginal outlet in some cases [1, 2].In $90 \%$ of the cases reported, rupture of the rudimentary horn occurred mostly in the second trimester [3]. Despite improvements in the diagnosis and treatment of cornual pregnancy, the majority is diagnosed following uterine rupture and the morbidity associated with this condition remains high [4].

The aim of this report is to present a case of rupture non-communicating rudimentary horn of a unicornuate uterus in a woman at 27 weeks of gestation, highlighting the importance of ultrasound and MRI in the diagnosis and the surgical management.

\section{Patient and Observation}

A 31-year-old woman, gravida 2 para 1, was pregnant at 27 weeks period of amenorrhea (POA). She hadn't medical or surgical history, but had a past obstetric history of postterm delivery at 41 weeks period of amenorrhea (POA). She delivered a $4000 \mathrm{~g}$ stillborn girl vaginally without any other complications. The patient presented with severe abdominal pain 4 hours prior to admission.

No other significant history was noted.

On examination, she was afebrile, looked ill, pale, with a pulse of 90 beats/minute and a blood pressure of $110 / 70 \mathrm{~mm}$ of $\mathrm{Hg}$. The abdomen was tense with marked tenderness, with a fundal height of $27 \mathrm{~cm}$ and soft uterine fundus. Per vaginum examination revealed a single cervix with an intact cervical canal. There was no active vaginal bleeding.

Abdominal and transvaginal ultrasounds revealed a single live pregnancy measuring approximately 26 weeks gestation with a posterior placenta; and an empty uterus which had a thickened endometrium. No apparent communication occurred between the gestational sac, and the cervix. The 
differential diagnosis at this time broadened to include a cornual or interstitial pregnancy and an abdominal pregnancy.

A MR Imaging of the abdomen and pelvis showed a well-defined gestational sac measuring $17 \mathrm{~cm} \times 13 \mathrm{~cm}$ with a single viable fetus in the rudimentary horn on the right side (Fig 1). A thin rim of myometrium could be identified around the gestational sac confirmed the extrauterine pregnancy. There was a moderate free fluid in peritoneal cavity. The remainder of the pelvic and abdominal anatomy was normal.

Laboratory tests on admission found hemoglobin $11.6 \mathrm{~g} /$ $\mathrm{dl}$, a second assessment carried out 12 hours after admission found hemoglobin of $9.7 \mathrm{~g} / \mathrm{dl}$, and despite the patient is stable hemodynamically except for a tachycardia at $100 \mathrm{bpm}$, the patient was taken for explorative laparotomy. Two liters of hemoperitoneum was removed intra-operatively, and the diagnosis of unicornuate uterus with ruptured right rudimentary horn pregnancy was confirmed. The rupture measured $2 \mathrm{~cm}$ at the fundus of non-communicating rudimentary horn with the externalization of placenta through it. The rudimentary horn was connected to the main uterus with a thick fibrous band (Fig 2, 3). There was no communication with the main uterine cavity. The right ovary appeared normal and the tube was normally attached to the rudimentary horn. The left adnexa appeared healthy.

A live born male fetus of $600 \mathrm{~g}$ was delivered with Apgar scores of 2 points at 1 and $5 \mathrm{~min}$, and unfortunately died after one hour.

A rudimentary horn excision, ipsilateral salpingectomy, and peritoneal toileting were carried out. The patient received five units of blood transfusion intra-operatively.

Macroscopic examination showed the placenta externalized through the fundic rupture (Fig 4). Histological examination revealed that the horn was blind and the placenta was increta. Patient had an uneventful recovery was discharged on the fifth post-operative day.

\section{Discussion}

A non-communicating rudimentary horn is a rare type of Mullerian duct malformation. The occurrence of pregnancy in a rudimentary horn is an extremely rare situation. The incidence is estimated from $1 / 100000$ to $1 / 140000$ pregnancies [5]. Pregnancy in unicornuate uterus with a non-communicating rudimentary horn is possible when transperitoneal migration of either spermatozoa or fertilized ovum from the contralateral side occurs [6].

Pregnancy continues for a variable period of time because of the varied muscular constitution in the thickness and distensibility of the wall in the rudimentary horn.

The pain associated with rudimentary horns in pregnancy commences from the end of the first and beginning of the second trimester. Vaginal bleeding is uncommon unless the horn is communicating [2].

The endometrium of rudimentary horn has been described as thinner than usual and sometimes nonfunctional leading to pathologic placentation leading to placenta accreta. The thin muscular wall of pregnant horn with presence of placenta accreta further increases the risk of rupture, during the second or third trimester, resulting in life-threatening hemoperitoneum like the case of our patient. Early diagnosis is the key to successful management of rudimentary uterine horn pregnancy.

Ultrasonographic criteria suggested by Tsafrir et al for the diagnosis of rudimentary horn pregnancy are a pseudopattern of an asymmetrical bicornuate uterus with absence of visual continuity of tissue surrounding the gestational sac and uterine cervix and presence of myometrial tissue around the gestational sac [7] as it was seen in our case. Unlike abdominal pregnancies, rudimentary horn pregnancies have a thin ring of myometrium that fits the placenta into the confines of the uterine horn, making it more identifiable [8].

In case of suspicion of rudimentary uterine horn pregnancy, magnetic resonance imaging (MRI) has proven to be a useful tool, both for the diagnosis and for the choice of surgical approaches [7].

An MRI examination is also helpful to determine whether any urinary tract abnormalities are present.

Placenta accreta accompanies rudimentary horn pregnancies in $13.7 \%$ of cases. Placentation problems are more likely to occur in rudimentary horn pregnancies than intrauterine pregnancies because the endometrium of a rudimentary uterine horn is thinner and less functional. This causes inadequate decidualization, thus leading to placenta accreta. The underdevelopment and poor distensibility of the myometrium and the propensity for abnormal placentation both contribute to the risk of uterine rupture in these cases [8].

Because of the hemorrhagic risk due to placenta accreta and that of spontaneous uterine rupture due to the thinness of the myometrium, immediate surgical removal of a pregnant rudimentary uterine horn and the ipsilateral salpingectomy as soon as its diagnosis is carried out [9].

Leaving the fallopian tube exposed to the theoretical and actual risk of tubal pregnancy [10].

The traditional management of RHP is laparotomy. But, there are now other modern techniques for the management of rudimentary horn pregnancy like laparoscopic and medical treatment.

Electrosurgery (bipolar forceps, Harmonic scalpel or a stapling device) successfully excised the pregnancy via a laparoscopic approach. These instruments provided for a relatively bloodless surgery despite the increased vascularity of the pregnant uterine horns $[8,11]$. Edelman et al. used a morcellator, which required less operative time and eliminated the need for a posterior colpotomy or a larger suprapubic incision [12].

The use of feticide like potassium chloride and intramuscular methotrexate followed by laparoscopic excision of the rudimentary horn a few months later is reported in the literature [13]. 


\section{Conclusion}

Non-communicating rudimentary uterine horn pregnancy is a rare entity associated with life threatening consequences. Early diagnosis and early interventions will avoid maternal morbidity and mortality.

Despite advances in ultrasound technology, the antenatal diagnosis of a rudimentary horn pregnancy remains difficult. If ultrasonography remains inconclusive, the use of magnetic resonance imaging is suggested.

\section{Figures}

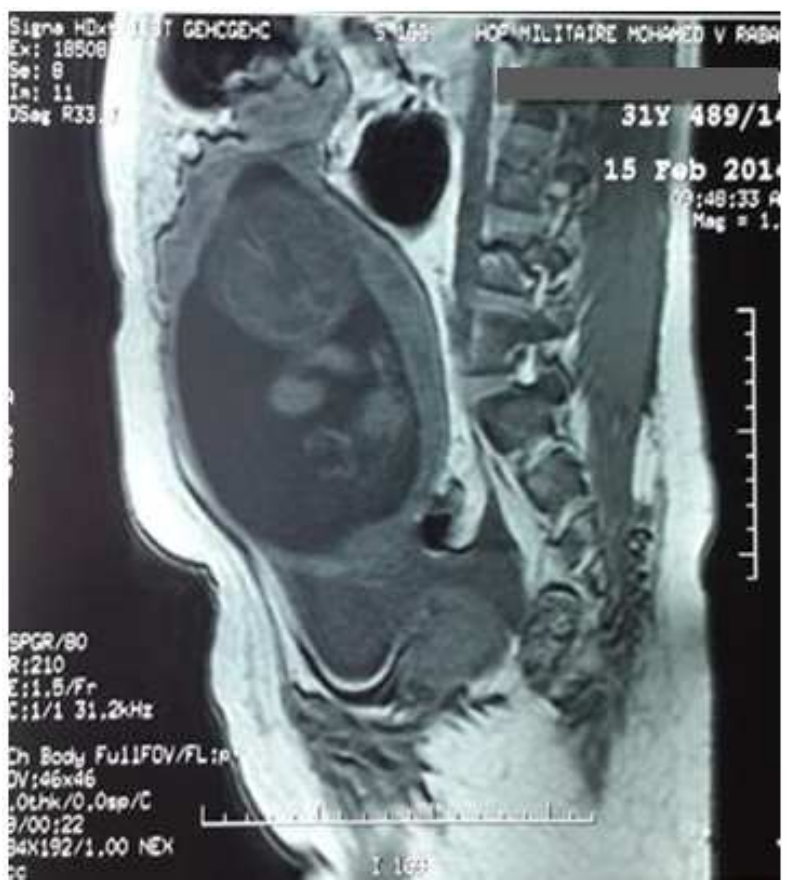

Figure 1. Imaging of abdomen showing non communicating rudimentary horn pregnancy

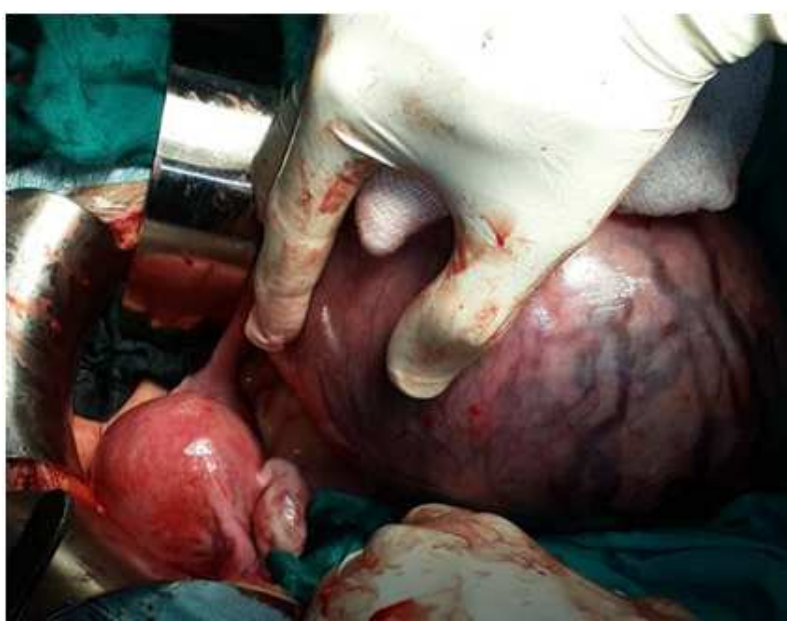

Figure 2. Intraoperative photographs showing the rudimentary uterine horn connected to the main uterus with a thick fibrous band

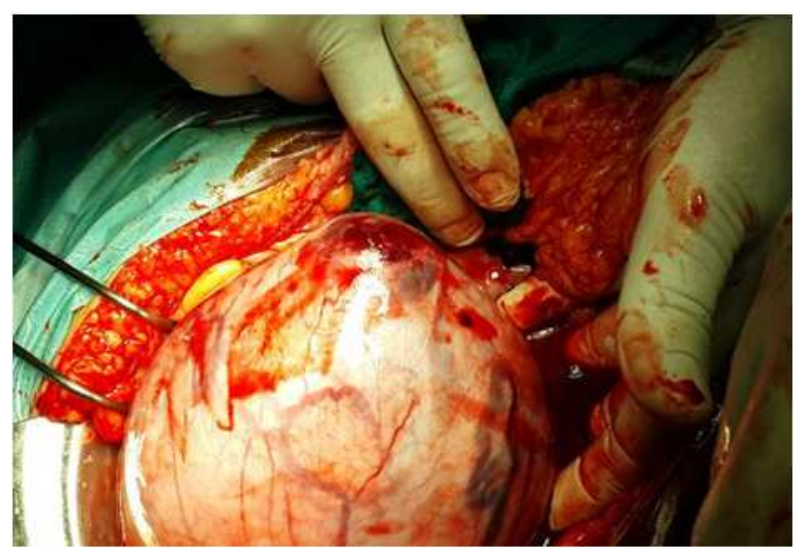

Figure 3. Intraoperative photographs showing the rudimentary uterine horn ruptured at the fundus

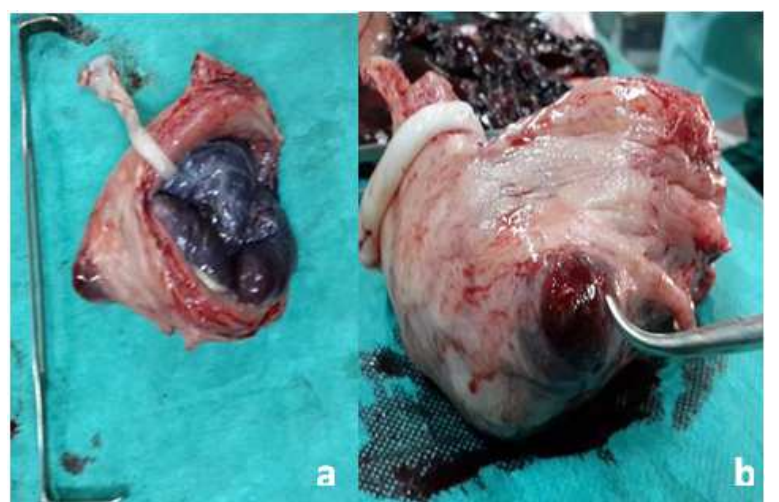

Figure 4 ( $\boldsymbol{a}$ and $\boldsymbol{b})$. Photographs showing the rudimentary horn with the placenta accreta externalized through the fundic rupture

\section{References}

[1] Dasari P. Pre-rupture ultrasound diagnosis of rudimentary horn pregnancy. Biomed research 2007;18(3):185-87.

[2] Upadhyaya, I. Non-communicating rudimentary uterine horn pregnancy. Journal of the Nepal Medical Association, 2011;51(4).

[3] Heinonen, P. K., \&Aro, P. Rupture of pregnant non communicating uterine horn with fetal salvage. European Journal of Obstetrics \& Gynecology and Reproductive Biology, 1988;27(3): 261-265.

[4] Nahum GG. Rudimentary uterine horn pregnancy.The $20^{\text {th }}$ century worldwide experience of 588 cases.J ReprodMed 2002; 47:151-163.

[5] Johansen K. Pregnancy in a rudimentary horn. ObstetGynecol 1983;61:565-7.

[6] Shinohara, A., A. Yamada, and A. Imai. "Rupture of noncommunicating rudimentary uterine horn at 27 weeks' gestation with neonatal and maternal survival." International Journal of Gynecology \& Obstetrics 2005; 88(3): 316-317.

[7] Tsafrir A, Rojansky N, Sela HY, Gomori JM, Nadjari M. Rudimentary horn pregnancy: first trimester prerupturesonographic diagnosis and confirmation by magnetic resonance imaging. $J$ Ultrasound Med $2005 ; 24: 219-23$. 
[8] Contreras, K. R., Rothenberg, J. M., Kominiarek, M. A., \& Raff, G. J. Hand-assisted laparoscopic management of a midtrimester rudimentary horn pregnancy with placenta increta: a case report and literature review. Journal of minimally invasive gynecology, 2008;15:644-648.

[9] Henriet E, Roman H, Zanati J, Lebreton B, Sabourin JC, Loic M. Pregnant noncommunicating rudimentary uterine horn with placenta percreta. JSLS. 2008;12:101-3.

[10] Heinonen P. Unicornuate uterus and rudimentary horn. FertilSteril 1997;68:224-30.
[11] So"nmezer M, Taskin S, Atabekoglu C, et al. Laparoscopic management of rudimentary uterine horn pregnancy: case report and literature review. JSLS. 2006;10:396-399.

[12] Edelman AB, Jensen JT, Lee DM, et al. Successful medical abortion of a pregnancy within a non-communicating uterine horn. Am J Obstet Gynecol. 2003;189:886-887.

[13] Cutner A, Saridogan E, Hart R, et al. Laparoscopic management of pregnancies occurring in a noncommunicating accessory uterine horns. Eur $\mathrm{J}$ Obstet Gynecol Reprod Biol. 2004;113:106-109. 